OSIPP OSIPP Discussion Paper : DP-2008-E-010

\title{
Entrepreneurial Competition and Its Impact on the Aggregate
}

\author{
Economy
}

September 3, 2008

\section{Katsuya Takii}

Associate Professor, Osaka School of International Public Policy (OSIPP)

【キーワード】 D21, D61, D83, L25, L26

【要約】This paper models entrepreneurship as the entrepreneur's information processing activity in order to predict changes in demand and reallocate resources. The results show that allocative efficiency---and therefore aggregate productivity--increases through intensified competition by entrepreneurs grasping at opportunities. This fierce competition leads to price reductions that result in the improvement of measured aggregate productivity. The price reduction also forces relatively less able entrepreneurs to become workers. As resources are then dealt with only by relatively talented entrepreneurs, this selection effect also increases aggregate productivity. The paper also discusses how the selection effect influences the distribution of firm size.

The financial support of the Japanese Ministry of Education, Culture, Sports, Science and Technology (Grant-in-Aid for Scientific Research) is gratefully acknowledged. Osaka School of International Public Policy, Osaka University, 1-31, Machikaneyama, Toyonaka, Osaka, 560-0043, Japan. E-mail: takii@osipp.osaka-u.ac.jp 


\section{Introduction}

Different researchers characterize entrepreneurs differently. It is well known that Knight (1921) emphasizes risk bearing as the main function of entrepreneurs while Schumpeter (1947) views entrepreneurs as persons who do new things. However, these are not the only functions of entrepreneurs discussed in the literature.

Many studies, for example, point out the prediction of idiosyncratic changes as an important element of entrepreneurship. Kirzner (1973) discusses the essential role of entrepreneurial discovery in market processes. Schultz (1975) defines entrepreneurial ability as the ability to interpret new information and reallocate resources to take advantage of profitable opportunities. More recently, Casson (2005) reviews the literature and argues that judgmental decision-making is a common feature of entrepreneurship.

Although these studies provide many valuable insights, they do not discuss how entrepreneurship influences aggregate statistics such as aggregate productivity and the distribution of firm size. These considerations demand a model to locate the prediction of idiosyncratic changes within standard macroeconomics.

This paper models entrepreneurship as the entrepreneur's information processing activity in order to predict changes in demand and reallocate resources. It then analyzes how entrepreneurship influences aggregate productivity. When there is uncertainty in demand, entrepreneurs reallocate their attention to processing information in order to predict the direction and magnitude of changes in demand. Although these changes can be inferred from a number of signals, some signals are more informative than others. Able entrepreneurs are assumed to know which signals are more informative for prediction. Guided by prior knowledge, they process specific signals in order to improve the accuracy of their predictions. Better prediction allows entrepreneurs to reallocate resources toward more profitable opportunities, and this 
improves the allocative efficiency of the economy.

The paper shows that the benefits of reallocation are captured by a reduction in an aggregate price index through fierce competition among firms. By processing local information, firms can increase their outputs when demand for their products is high, enabling them to set a higher price. Conversely, firms can reduce outputs when they set a lower price due to low demand. The strong correlation between price and output increases the firm's expected revenues. However, when total expenditure is constant, an increase in firm revenue involves a reduction in expenditure on goods provided by other firms. This demand-stealing effect results in a reduction in the aggregate price index and, therefore, an increase in measured aggregate productivity.

If ability differs across agents, competition leads to selection among entrepreneurs. This provides an additional mechanism that influences aggregate productivity. As the reduction in the aggregate price index lowers expected profits and increases real wage payments, less able entrepreneurs choose to be workers. Because more resources are then dealt with only by high-ability entrepreneurs in equilibrium, allocative efficiency and aggregate productivity in the economy again increase.

Note that this selection mechanism also influences the distribution of firm size. As the increase in the ability difference across entrepreneurs forces less able entrepreneurs to become workers, it reduces the number of firms and increases the number of available workers. Hence, it increases not only size differences across firms, but also the average firm size.

The role of entrepreneurial ability in the economy is magnified when demand is more volatile and goods more substitutable. An increase in idiosyncratic risk increases the importance of prediction, whereas an increase in the substitutability of goods makes the demand for each good more sensitive to changes in tastes. Hence, both increase the benefits of prediction. As the increase in risk and the substitutabil- 
ity of goods increases the benefits from more able entrepreneurs, they force less able entrepreneurs to be workers through a further reduction in the aggregate price. It is then shown that increases in risk and the substitutability of goods increase not only the average size differences, but also the average size of firms.

This paper is organized as follows. Section 2 reviews the literature and clarifies the concepts used in the paper. Section 3 sets up the model. Section 4 analyzes the equilibrium and derives real GDP as a function of entrepreneurship. Section 5 analyzes how a change in the distribution of entrepreneurial ability influences real GDP and the size distribution of firms. Section 6 concludes by summarizing the main results and discussing some possible extensions.

\section{Literature Review}

Labor economists empirically investigate the importance of the ability to predict changes. Welch (1970) points out that the effect of education on the production process can be decomposed into two components. Higher education enables a worker to increase the amount of output produced from a given quantity of inputs (the worker effect); it also allows the worker to interpret information about the profitability of resource allocation, and this enables the worker to make better use of resources (the allocative effect). Schultz (1975) regards the allocative effect of education as the source of entrepreneurial ability and Takii (2003) compares these effects of human intelligence in the context of managers' human capital: managerial ability is considered as the ability to organize and increase productivity and entrepreneurial ability is considered as the ability to predict and react changes. This study follows this distinction but allows entrepreneurs to process information based on knowledge about the relationship between observable information and shocks in taste. Hence, entrepreneurial ability is measured by the importance of knowledge for prediction. 
Another strand of the literature suggests that information processing activities can also be an important component of entrepreneurship [e.g., Fiet (1996) and Casson (2005)]. There are several features of entrepreneurial information processing: 1) By processing information, entrepreneurs observe risk-reducing signals [e.g., Fiet (1996)]. 2) Information must be specific [e.g., Hayek (1945), Fiet (1996) and Casson (2005)]. 3) Entrepreneurial discovery occurs under imperfect competition [e.g., Fiet (1996) and Kirzner (1997)]. 4) An entrepreneur is better suited to assess specific information because of appropriate prior knowledge, which can be the result of previous experience [e.g., Fiet (1996) and Shane (2000)] and/or education [e.g., Schultz (1975)]. This paper incorporates all of these features of entrepreneurship into a general equilibrium model.

Because this paper defines entrepreneurial ability as the entrepreneur's knowledge about the relationship between observable information and changes in demand, the analysis can be linked naturally to firm decisions under uncertainty. In the nice survey by Hey (1979), the previous literature on firm decisions under uncertainty takes information structure as given and analyzes its impact on firm's decision in a partial equilibrium model. In contrast, this paper assumes that entrepreneurship can convert information structure and analyzes how entrepreneurship influences the aggregate economy in a general equilibrium model.

It is also important to distinguish between differences in prediction accuracy and flexibility. Economic theories of the firm investigate how firms adapt to changes by examining firm flexibility. Many theorists investigate the tradeoff between static efficiency and flexibility (e.g., Mills and Schumann (1985) and Thesmar and Thoenig (2000)). Unlike these studies, we pay greater attention to a firm's prediction ability. In other words, we investigate the ability of firms to adapt appropriately to changes in demand, whereas the existing literature on flexibility investigates the ability of 
firms to quickly or costlessly adapt to changes in demand. As flexibility improves profits only when firms know how decisions should be changed, our results provide useful complementary information for making inferences about the importance of these theories.

This is not the first paper to incorporate a similar definition of entrepreneurial ability into a general equilibrium model [e.g., Hassler and Mora (2000), Takii (2004; 2008)]. Hassler and Mora (2000) discuss the relation to social mobility and Takii (2004; 2008) considers the impact of entrepreneurial ability on aggregate productivity and on expansionary fiscal policy, respectively. However, no earlier work explicitly models how entrepreneurs process information to change information structure, which is the essence of entrepreneurship in this paper, and no study examines the impact of entrepreneurship on the size distribution of firms.

\section{Model}

In this section, we construct a model to analyze the aggregate impact of entrepreneurship. There are two occupations in an economy: entrepreneurs and workers. Each firm needs one entrepreneur to organize the firm. In this model, for simplicity, there is no distinction between entrepreneurs, managers, plants, and firms. This simplification is made to develop a tractable model that focuses on information processing activity as the essence of entrepreneurship. The goods market is assumed to be monopolistically competitive and each entrepreneur faces her own inverse demand function and produces output by employing workers. Each demand curve is subject to taste shocks that entrepreneurs cannot observe when they make production decisions. They process information and infer the direction and magnitude of these taste shocks. We construct a model to analyze how these information processing activities influence the aggregate economy. 
Inverse Demand Function: There are heterogeneous households in an economy. The $j$ th household is assumed to maximize the following utility subject to its budget constraint:

$$
\begin{aligned}
U_{j} & \equiv \max _{x_{i j}}\left[\int_{i}^{m} z_{i} x_{i j}^{\alpha} d i\right]^{\frac{1}{\alpha}}, \alpha=1-\frac{1}{\rho} \in(0,1), \\
\text { s.t. } Y_{j}^{n} & =\int_{i}^{m} p_{i} x_{i j} d i,
\end{aligned}
$$

where $x_{i j}$ is the amount of good $i$ consumed by the $j$ th household, $z_{i}$ is a productspecific taste shock for product $i, p_{i}$ is the price for product $i, m$ is the number of products, and $Y_{j}^{n}$ is the expenditure of the $j$ th household. As the parameter $\rho$ is the measure of the substitutability of goods, $\alpha$ also captures the substitutability of goods. Using standard procedures, the inverse demand function for good $i$ can be derived as follows.

$$
\begin{aligned}
p_{i} & =z_{i} x_{i}^{\alpha-1} P^{\alpha}\left(Y^{n}\right)^{1-\alpha}, \\
P & =\left[\int_{i}^{m} p_{i}^{\frac{\alpha}{\alpha-1}} z_{i}^{\frac{1}{1-\alpha}} d i\right]^{\frac{\alpha-1}{\alpha}}, \\
Y^{n} & =\int Y_{j}^{n} d j, x_{i}=\int x_{i j} d j,
\end{aligned}
$$

where $P$ is the price index and $Y^{n}$ is nominal GDP. As we assume that the household sector is the only sector that buys products, the total sum of consumer expenditure is nominal GDP in this analysis.

A Firm's Problem: An entrepreneur establishes a firm, employs workers, and produces output. A single firm produces one variety of each good and an entrepreneur faces an inverse demand curve, equation (1). However, when she undertakes production, she does not know what the realized taste shock is. The timing sequence 
is described as follows. (1) A taste shock occurs. (2) An entrepreneur processes this information and infers the realized taste shock. (3) A firm produces nonstorable goods by choosing the amount of employed workers. (4) The firm discovers the true taste shock. (5) The firm sets its price to equate demand with the goods produced. We now describe the behavior of a representative firm and omit subscript $i$ where the intended meaning is clear.

Because the movement of $z$ is not observed ex ante when $z$ changes, the entrepreneur must predict the direction and magnitude of the change so as to appropriately respond. When the entrepreneur makes production decisions, $z$ is not observed, but noisy signals are observed, $\mathbf{S}^{A v} \equiv\left\{s_{j}^{A}\right\}_{j=1}^{v}$, from which the realization of $z$ can be inferred. As discussed later, the accuracy of prediction depends on the quality of signals, $A$, and the amount of signals, $v$, the entrepreneur actually processes. We consider that the variable $v$ captures the intensity of entrepreneurship. It is assumed that the entrepreneur's inference is based on a conditional distribution function, $Q^{h}\left(z \mid \mathbf{S}^{A v}\right)$, where $h$ measures the accuracy of an entrepreneur's prediction for changes in $z$. We specify the information structure later.

Note that because $z$ is specific to a particular product, the required information is also specific to the product. As only one firm produces this product, this is purely firm-specific information. Many researchers point out the importance of specific information in the entrepreneurial discovery process. The current model incorporates the spirit of this argument.

The production function is assumed to be linear: $x=M(v) L$ in labor where $L$ is the amount of labor input and $M(v)$ is the productivity of a firm's production process. When an entrepreneur establishes a firm, she must set up the production process in order to organize the firm. This activity is assumed to increase the productivity of a firm's production process. When an entrepreneur processes more signals, $v$, she 
may not be able to spend enough time setting up the production process. Hence, we assume that $M^{\prime}(v)<0$.

It is assumed that the financial market is complete. Therefore, entrepreneurs can hedge any idiosyncratic risk. Although financial investors cannot scrutinize the information entrepreneurs observe, they are assumed to know the entrepreneurs' effort level and their ability, which influence the accuracy of information, $h$. Hence, there is no distortion caused by asymmetric information. Entrepreneurs maximize their firm's expected profits:

$$
\begin{aligned}
\pi\left(\mathbf{S}^{A v}\right) & =\max _{L}\left\{\int p d Q^{h}\left(z \mid \mathbf{S}^{A v}\right) x-w L\right\} \\
\text { s.t. } p & =z x^{\alpha-1} P^{\alpha}\left(Y^{n}\right)^{1-\alpha}, x=M(v) L
\end{aligned}
$$

where $w$ is the wage rate.

The solution to the profit maximization problem reveals that the optimal price, $p\left(z, \mathbf{S}^{A v}\right)$, has to be equal to a constant markup, $\frac{1}{\alpha}$, times marginal cost, $\frac{w}{M(v)}$, on average:

$$
p\left(z, \mathbf{S}^{A v}\right)=\frac{z}{\int z Q^{h}\left(z \mid \mathbf{S}^{A v}\right)} \frac{w}{\alpha M(v)} .
$$

Note that the actual price deviates from the constant markup rule due to entrepreneurs misunderstanding changes in tastes, $\frac{z}{\int z Q^{h}\left(z \mid \mathbf{S}^{A v}\right)}$. When the true $z$ is discovered, the entrepreneur realizes that it differs from the predicted value. If the realized $z$ is greater than the predicted one, $\int z Q^{h}\left(z \mid \mathbf{S}^{A v}\right)$, then the entrepreneur can set a higher price than the markup rule. If $z$ is lower than $\int z Q^{h}\left(z \mid \mathbf{S}^{A v}\right)$, then the entrepreneur must set a lower price.

Entrepreneurs produce more when they can set high prices, and produce less when they must set low prices. Hence, expected revenue is influenced by the accuracy of 
prediction:

$$
\begin{aligned}
E^{h}[p x] & =\left[z(h) M(v)^{\alpha}\right]^{\frac{1}{1-\alpha}}\left[\frac{\alpha P}{w}\right]^{\frac{\alpha}{1-\alpha}} Y^{n}, \\
z(h) & \equiv\left[\int\left[\int z d Q^{h}\left(z \mid \mathbf{S}^{A v}\right)\right]^{\frac{1}{1-\alpha}} d Q_{s}^{h}\left(\mathbf{S}^{A v}\right)\right]^{1-\alpha},
\end{aligned}
$$

where $E^{h}[p x]=\int p\left(z, \mathbf{S}^{A v}\right) x\left(\mathbf{S}^{A v}\right) d Q_{z s}^{h}\left(z, \mathbf{S}^{A v}\right), Q_{s}^{h}\left(\mathbf{S}^{A v}\right)$ and $Q_{z s}^{h}\left(z, \mathbf{S}^{A v}\right)$ is the marginal and joint distribution function of $z$ and $S^{A v}$.

Furthermore, as the expected amount of labor and the expected profits are proportional to $E^{h}[p x]$ in this model, the accuracy of prediction also influences them through its impact on expected revenue.

$$
\begin{aligned}
\int L\left(\mathbf{S}^{A v}\right) d Q_{s}^{h}\left(\mathbf{S}^{A v}\right) & =\frac{\alpha}{w} E^{h}[p x], \\
\int \pi\left(\mathbf{S}^{A v}\right) d Q_{s}^{h}\left(\mathbf{S}^{A v}\right) & =(1-\alpha) E^{h}[p x],
\end{aligned}
$$

where $L(\cdot)$ is an optimal labor function.

Information Structure: The accuracy of information can be a function of entrepreneurship. We assume that entrepreneurs use their ability to process information and make sound judgments. In order to construct an operational model, we must specify the information structure. Assume that $\ln z$ is normally distributed with mean $-\frac{\sigma_{z}^{2}}{2}$ and variance $\sigma_{z}^{2}$. This means that the expected value of $z$ is normalized at 1 . The random deviation of $z$ from 1 represents ex ante unknown changes in taste.

Assume that there are many signals that are correlated with $\ln z$ with error:

$$
s_{j}^{A}=\ln z+\varepsilon_{j}^{A}
$$

where $\varepsilon_{j}^{A}$ is normally distributed with mean 0 and variance $\frac{\sigma_{z}^{2}}{A}$. Signals differ by their precision. People may learn the relationship between a signal, $s_{j}^{A}$, and shock, 
$\ln z$, from education or previous experience. But without knowing this relationship, signals are of no value to entrepreneurs. Researchers are split on their opinions about how prior knowledge is obtained. However, all agree that entrepreneurs are heterogeneous in terms of the knowledge used to detect important information. Talented entrepreneurs are assumed to know which signal is more accurate. Hence, the parameter $A$ can be interpreted as the measure of entrepreneurial ability.

Using their prior knowledge, entrepreneurs observe this signal $v$ times. Let us measure the accuracy of prediction by $h=1-\frac{\int\left(\ln z-\int \ln z d Q^{h}\left(z \mid \mathbf{S}^{A v}\right)\right)^{2} d Q_{z s}^{h}\left(z, \mathbf{S}^{A v}\right)}{\sigma_{z}^{2}}$, which is suggested by Takii (2007) as an estimable measure of the accuracy of prediction. This measure is equivalent to $R^{2}$ that is obtained when we regress $\ln z$ on $\frac{\sum_{j}^{v} s_{j}^{A}}{v}$. It is easy to show that $h$ can be a function of $A$ and $v:^{1}$

$$
h=\eta(A v) \equiv \frac{A v}{A v+1} .
$$

This shows that the intensity of entrepreneurship, $v$, and entrepreneurial ability, $A$, increase $h$ and, therefore, reduce perceived risk. This is consistent with arguments by Fiet (1996). Applying Lemma 2 in Takii (2007) to the current problem, $z(h)$ is shown to be a function of $A$ and $v$.

$$
z(h)=\exp \frac{\alpha \sigma_{z}^{2} \eta(A v)}{2(1-\alpha)} .
$$

\footnotetext{
${ }^{1}$ The measure $h$ can be rewritten as $h=\frac{\int\left[\int \ln z d Q^{h}\left(z \mid \mathbf{S}^{A v}\right)-\int \ln z d Q_{z}^{h}(z)\right]^{2} d Q_{s}^{h}\left(\mathbf{S}^{A v}\right)}{\sigma_{z}^{2}}$. This analysis focuses on a multiplicative shock, and the shock and the corresponding signal are log-normally distributed. In this case, we can show that signals are informative in the sense of Blackwell (1953) if and only if the conditional expectation $\int \ln z d Q^{h}\left(z \mid \mathbf{S}^{A v}\right)$ is risky in the sense of Rothschild and Stiglitz (1970). The measure $h$ captures this property. Of course, the variation of $\ln z$ can also influence that of $\int \ln z d Q^{h}\left(z \mid \mathbf{S}^{A v}\right)$. Hence, we divide it by $\sigma_{z}^{2}$ so that $h$ is independent of $\sigma_{z}^{2}$. Hence, $h$ is not influenced by exogenous risk.
} 
Entrepreneurship: Processing information reduces the time available to set up the production process due to their limited attention capacity. Hence, it lowers the productivity of their firm's production process. Knowing this tradeoff, entrepreneurs optimally choose the amount of information they observe. We assume that $M(v)=\exp (-\beta v)$ for a simple analysis. An entrepreneur's decision problem is then summarized as follows.

$$
\begin{aligned}
\Pi(A) & \equiv \max _{v}(1-\alpha) E^{\eta(A v)}[p x] \\
E^{\eta(A v)}[p x] & =\left[\exp \frac{\alpha x\left(\alpha, \sigma_{z}^{2}, A, v\right)}{1-\alpha}\right]\left[\frac{\alpha P}{w}\right]^{\frac{\alpha}{1-\alpha}} Y^{n}, \\
x\left(\alpha, \sigma_{z}^{2}, A, v\right) & =\frac{\sigma_{z}^{2} \eta(A v)}{2(1-\alpha)}-\beta v .
\end{aligned}
$$

Define $v\left(\alpha, \sigma_{z}^{2}, A\right)$ as the optimal solution to this problem. Ignoring integer constraints, we assume that the first-order conditions can approximate optimal conditions. The optimal conditions can then be described as follows.

$$
\begin{aligned}
& v\left(\alpha, \sigma_{z}^{2}, A\right)=\frac{\sqrt{\frac{\sigma_{z}^{2} A}{2 \beta(1-\alpha)}}-1}{A}, \text { if } A \geq \frac{2 \beta(1-\alpha)}{\sigma_{z}^{2}}, \\
& v\left(\alpha, \sigma_{z}^{2}, A\right)=0, \text { if } A<\frac{2 \beta(1-\alpha)}{\sigma_{z}^{2}} .
\end{aligned}
$$

This shows that if entrepreneurs do not have enough prior knowledge, they choose not to process information. Instead, they focus on preparation for the production process so that they can produce more. However, the impact of ability on the amount of processing information is not monotonic. It is shown that

$$
\begin{aligned}
& v_{A}\left(\alpha, \sigma_{z}^{2}, A\right) \geq 0, \text { if } \frac{2 \beta(1-\alpha)}{\sigma_{z}^{2}} \leq A \leq \frac{8 \beta(1-\alpha)}{\sigma_{z}^{2}} . \\
& v_{A}\left(\alpha, \sigma_{z}^{2}, A\right)<0, \text { if } A>\frac{8 \beta(1-\alpha)}{\sigma_{z}^{2}}
\end{aligned}
$$

When entrepreneurs know where an informative signal is, they have greater incentive to process the information. However, if signals are extremely accurate, entrepreneurs 
do not require too many signals. For example, if entrepreneurs can perfectly observe the movement of $\ln z$, just one observation is sufficient to detect the movement in $\ln z$.

Note that increases in the substitutability of goods and risk increase the amount of information that entrepreneurs process:

$$
v_{\alpha}\left(\alpha, \sigma_{z}^{2}, A\right)>0, v_{\sigma_{z}^{2}}\left(\alpha, \sigma_{z}^{2}, A\right)>0, \text { if } A \geq \frac{2 \beta(1-\alpha)}{\sigma_{z}^{2}}
$$

An increase in risk increases the value of accurate information, while an increase in the substitutability of goods makes demand more sensitive to taste shocks. Hence, this also demands more information.

It is interesting to note that the substitutability of goods increases flexibility in changing the amount of output. When goods are more substitutable, equation (1) suggests that the demand curve is flatter. Hence, an entrepreneur can change the amount of output without reducing prices greatly. An entrepreneur can exploit the benefits from better information when she can easily change her decisions. Hence, an entrepreneur spends more time processing information when $\alpha$ is large.

Although an increase in entrepreneurial ability has ambiguous effects on the amount of information processed, it unambiguously increases profits. To see this, let us define a function $x^{*}$ such that $x^{*}\left(\alpha, \sigma_{z}^{2}, A\right) \equiv x\left(\alpha, \sigma_{z}^{2}, A, v\left(\alpha, \sigma_{z}^{2}, A\right)\right)$. Then $x^{*}\left(\alpha, \sigma_{z}^{2}, A\right)$ can be solved as

$$
\begin{aligned}
x^{*}\left(\alpha, \sigma_{z}^{2}, A\right) & =\frac{\beta}{A}\left[\sqrt{\frac{\sigma_{z}^{2} A}{2 \beta(1-\alpha)}}-1\right]^{2}, \text { if } A \geq \frac{2 \beta(1-\alpha)}{\sigma_{z}^{2}} \\
& =0, \text { if } A<\frac{2 \beta(1-\alpha)}{\sigma_{z}^{2}}
\end{aligned}
$$


We can easily check that when $A \geq \frac{2 \beta(1-\alpha)}{\sigma_{z}^{2}}$,

$$
\begin{aligned}
x_{A}^{*}\left(\alpha, \sigma_{z}^{2}, A\right) & >0, \\
x_{\alpha}^{*}\left(\alpha, \sigma_{z}^{2}, A\right) & >0, x_{\sigma_{z}^{2}}^{*}\left(\alpha, \sigma_{z}^{2}, A\right)>0, \\
x_{A \alpha}^{*}\left(\alpha, \sigma_{z}^{2}, A\right) & >0, x_{\sigma_{z}^{2} A}^{*}\left(\alpha, \sigma_{z}^{2}, A\right)>0 .
\end{aligned}
$$

Equation (7) shows that entrepreneurial ability increases profits. In addition, equation (8) and equation (9) show that not only are profits increasing in $\alpha$ and $\sigma_{z}^{2}$, but $\alpha$ and $\sigma_{z}^{2}$ are also complementary in $A$. That is, increases in risk and the substitutability of goods increase the marginal profitability of entrepreneurial activities.

Let us define a function $\eta^{*}$ such that $\eta^{*}(A)=\eta\left[A v\left(\alpha, \sigma_{z}^{2}, A\right)\right]$. Because entrepreneurial ability increases firm prediction, expected revenue is an increasing function of $A$ :

$$
E^{\eta^{*}(A)}[p x]=\exp \left[\frac{\alpha x^{*}\left(\alpha, \sigma_{z}^{2}, A\right)}{1-\alpha}\right]\left[\frac{\alpha P}{w}\right]^{\frac{\alpha}{1-\alpha}} Y^{n} .
$$

Note that $\exp \left[\frac{\alpha x^{*}\left(\alpha, \sigma_{z}^{2}, A\right)}{1-\alpha}\right]=M\left(v\left(\alpha, \sigma_{z}^{2}, A\right)\right)=1$ if and only if $v\left(\alpha, \sigma_{z}^{2}, A\right)=0$. Hence, the component $\exp \left[\frac{\alpha x^{*}\left(\alpha, \sigma_{z}^{2}, A\right)}{1-\alpha}\right]=\frac{E^{\eta^{*}(A)}[p x]}{E^{0}[p x]} \geq 1$ is the relative expected revenue gain from processing information. In this paper, this is considered as the gain from entrepreneurship. Entrepreneurship improves expected revenue because it allows entrepreneurs to produce more when demand is high and less when demand is low. As shown, this revenue gain is an increasing in $\alpha, \sigma_{z}^{2}$ and $A$.

The arbitrage condition: An agent can be an entrepreneur or a worker. Let us assume that entrepreneurial ability is distributed along a continuous distribution function $G(A)$. Let $N$ denote the number of workers that have an ability less than or equal to $A$. Then $G(A)=N$. It is convenient to define an inverse distribution function $\Gamma$ such that $A=\Gamma(G(A))$. The use of the inverse distribution function makes it easier to analyze the model. The value $\Gamma(N)$ represents the ability level 
that the $\frac{N}{N^{s}}$ percentile from bottom agent obtains, where $N^{s}$ is the total population.

Because entrepreneurs can completely hedge their risks in the financial market, they do not bear risk. As $x_{A}^{*}\left(\alpha, \sigma_{z}^{2}, A\right) \geq 0, \Pi^{\prime}(A) \geq 0$. Because $G(A)$ is continuous distribution function, $\Gamma^{\prime}(\cdot)>0$. Hence, there is a unique cutoff ability $\Gamma\left(N^{*}\right)$ with which an agent expects to earn the same income from being an entrepreneur or a worker:

$$
w=\Pi\left(\Gamma\left(N^{*}\right)\right)=(1-\alpha) E^{\eta^{*}\left(\Gamma\left(N^{*}\right)\right)}[p x] .
$$

The second equation is derived because the expected profits are proportional to the expected revenue.

Resource constraints: Using the function $\Gamma$ we can define the labor market clearing condition as follows.

$$
N^{*}=\int_{N^{*}}^{N^{s}} \int L\left(\mathbf{S}^{A v}\right) d Q_{s}^{\eta^{*}(\Gamma(N))}\left(\mathbf{S}^{A v}\right) d N
$$

The left-hand side is the supply of labor and the right-hand side is the demand for labor. The equation states that the bottom $N^{*}$ agents in the ability distribution become workers and the top $N^{s}-N^{*}$ agents in the ability distribution become entrepreneurs. As entrepreneurs with the ability $\Gamma(N)$ demand labor by $\int L\left(\mathbf{S}^{A v}\right) d Q_{s}^{\eta^{*}(\Gamma(N))}\left(\mathbf{S}^{A v}\right)$, the right-hand side of equation (12) is the total demand for labor.

As expected labor is proportional to expected revenue in equation (5), equation (12) can be rewritten as

$$
N^{*}=\frac{\alpha}{w} \int_{N^{*}}^{N^{s}} E^{\eta^{*}(\Gamma(N))}[p x] d N
$$




\section{The Distribution of Firm and Real GDP}

In this section, we investigate the implications for the distribution of firm size and real GDP. First, we show the existence and uniqueness of equilibrium and discuss the implications on the distribution of firm size. Later, we derive real GDP and discuss the effect of entrepreneurial ability on aggregate productivity.

Equilibrium: This is derived from equation (11) and (13) where

$$
\int_{N^{*}}^{N^{s}} \exp \frac{\alpha\left[x^{*}\left(\alpha, \sigma_{z}^{2}, \Gamma(N)\right)-x^{*}\left(\alpha, \sigma_{z}^{2}, \Gamma\left(N^{*}\right)\right)\right]}{1-\alpha} d N=\frac{1-\alpha}{\alpha} N^{*}
$$

This equation determines an equilibrium level of $N^{*}$. The existence and uniqueness of $N^{*}$ can be easily proved. The formal proof is established in the Appendix.

Theorem 1 There exists a unique $N^{*}$.

Note that $N^{*}$ contains several items of information. First, $N^{*}$ is the number of workers. Second, $N^{s}-N^{*}$ is the number of firms. Third, $\frac{N^{*}}{N^{s}-N^{*}}$ can be interpreted as the average firm size. Fourth, it also relates to the average differences in revenue. As $\exp \left[\frac{\alpha x^{*}\left(\alpha, \sigma_{z}^{2}, A\right)}{1-\alpha}\right]=\frac{E^{\eta^{*}(A)}[p x]}{E^{0}[p x]}$, the expected revenue differences due to differences in entrepreneurial ability are captured by $\exp \frac{\alpha\left[x^{*}\left(\alpha, \sigma_{z}^{2}, \Gamma(N)\right)-x^{*}\left(\alpha, \sigma_{z}^{2}, \Gamma\left(N^{*}\right)\right)\right]}{1-\alpha}$. Hence, the average revenue differences across firms can be measured by $\frac{\int_{N^{*}}^{N^{s}} \exp \frac{\alpha\left[x^{*}\left(\alpha, \sigma_{z}^{2}, \Gamma(N)\right)-x^{*}\left(\alpha, \sigma_{z}^{2}, \Gamma\left(N^{*}\right)\right)\right]}{1-\alpha} d N}{N^{s}-N^{*}}$. Equation (14) suggests that $\frac{1-\alpha}{\alpha} \frac{N^{*}}{N^{s}-N^{*}}$ is equivalent to the average revenue differences across firms.

Note that the average revenue differences can also be interpreted as the average size differences. Equations (5) and (6) imply that the expected revenue differences are translated into differences in expected labor cost and differences in expected profits. 
Hence, it is natural to interpret $\frac{\int_{N^{*}}^{N^{s}} \exp \frac{\alpha\left[x^{*}\left(\alpha, \sigma_{z}^{2}, \Gamma(N)\right)-x^{*}\left(\alpha, \sigma_{z}^{2}, \Gamma\left(N^{*}\right)\right)\right]}{1-\alpha} d N}{N^{s}-N^{*}}$ and, therefore, $\frac{1-\alpha}{\alpha} \frac{N^{*}}{N^{s}-N^{*}}$, as a measure of average size differences.

This interpretation suggests that the average firm size and the average size differences move together with $N^{*}$ in the equilibrium. Applying the implicit function theorem, we can show how the changes in $\alpha$ and $\sigma_{z}^{2}$ influence $N^{*}$. The formal proof is established in the Appendix.

Proposition 1 There is a function $N$ such that

$$
N^{*}=N\left(\alpha, \sigma_{z}^{2} ; \Gamma\right), N_{\alpha}>0, N_{\sigma_{z}^{2}}>0
$$

This proposition implies that if goods are substitutable and the economy is risky, the number of workers is large, the average firm size is large, and the average size differences across firms are large.

This is because ability is complementary to risk and the substitutability of goods. A risky environment demands more accurate information. An increase in risk then increases the importance of prediction, whereas an increase in the substitutability of goods makes demand more sensitive to changes in tastes. Hence, they both increase the benefits of able entrepreneurs and less able entrepreneurs are forced to be workers. This results in an increase in the average firm size and the average size differences across firms.

It is interesting to note that an increase in risk may increase size. The standard argument emphasizes the tradeoff between static efficiency and flexibility under uncertain demand. As efficiency is easily converted to size and a risky environment demands flexibility, there is a tradeoff between size and risk [e.g., Mills and Schumann (1985)]. In fact, it would be possible to extend our model to derive a negative relationship between risk and size by introducing irreversible investment. In this sense, we cannot take this result as an empirically testable prediction. Rather, this 
proposition suggests that there is a neglected mechanism where risk increases size. The relationship between risk and size may then be more complex than conventional theories suggest.

Real GDP: In order to understand the impact on productivity, we derive real GDP in this model. Note that the definition of nominal GDP implies

$$
Y^{n}=\int_{N^{*}}^{N^{s}} E^{\eta^{*}(\Gamma(N))}[p x] d N .
$$

Substituting equation (15) into equation (13), nominal GDP is shown to be proportional to the total labor cost:

$$
Y^{n}=\frac{w N^{*}}{\alpha}
$$

In order to derive real GDP, we require an aggregate price index. Substituting equation (10) into (15), the aggregate price index is shown to decline as a result of entrepreneurship in the economy.

$$
\begin{aligned}
P & =\frac{w}{\alpha z^{*}}, \\
z^{*} & =\left[\int_{N^{*}}^{1} \exp \frac{\alpha x\left(\alpha, \sigma_{z}^{2}, \Gamma(N)\right)}{1-\alpha} d N\right]^{\frac{1-\alpha}{\alpha}} .
\end{aligned}
$$

Note that $P$ is decreasing in $z^{*}$, which is a positive function of the total sum of the relative expected revenue gain due to entrepreneurship. When all entrepreneurs process information, they can increase revenue on average. However, total expenditure is given by $Y^{n}$. This means that an increase in the revenue of a particular firm results in the reduction of expenditure on other firms, bringing about a reduction in the aggregate price index.

Dividing nominal GDP by the aggregate price index, real GDP can be expressed as a function of the number of workers and $z^{*}$ :

$$
Y=\frac{Y^{n}}{P}=z^{*} N^{*}
$$


This equation shows that $z^{*}$ also represents the improvement in aggregate productivity due to entrepreneurship. Entrepreneurship increases aggregate productivity because it improves the allocative efficiency of the economy. Because entrepreneurship allows managers to provide output when needed, it also improves social welfare. As shown when $Y=\int U_{j} d j$, the improvement in social welfare is captured by the increase in real GDP.

Aggregate productivity $z^{*}$ can be decomposed into a level effect and a distributional effect:

$$
\begin{aligned}
z^{*} & =\text { Dist } \times \text { Level }, \\
\text { Dist } & =\left(\int_{N^{*}}^{N^{s}} \exp \frac{\alpha\left[x^{*}\left(\alpha, \sigma_{z}^{2}, \Gamma(N)\right)-x^{*}\left(\alpha, \sigma_{z}^{2}, \Gamma\left(N^{*}\right)\right)\right]}{1-\alpha} d N\right)^{\frac{1-\alpha}{\alpha}}, \\
\text { Level } & =\exp x^{*}\left(\alpha, \sigma_{z}^{2}, \Gamma\left(N^{*}\right)\right) .
\end{aligned}
$$

If there is no heterogeneity across agents, everybody has the same ability, $\Gamma\left(N^{*}\right)$. Hence, only the level effect, $\exp x^{*}\left(\alpha, \sigma_{z}^{2}, \Gamma\left(N^{*}\right)\right)$, influences the productivity. It implies that $\exp x^{*}\left(\alpha, \sigma_{z}^{2}, \Gamma(N)\right)$ can be interpreted as the productivity gain induced by ability $\Gamma(N)$. Then, the distribution effect, $\left(\int_{N^{*}}^{N^{s}} \exp \frac{\alpha\left[x^{*}\left(\alpha, \sigma_{z}^{2}, \Gamma(N)\right)-x^{*}\left(\alpha, \sigma_{z}^{2}, \Gamma\left(N^{*}\right)\right)\right]}{1-\alpha} d N\right)^{\frac{1-\alpha}{\alpha}}$, can be interpreted as the weighted sum of productivity differences across firms. This summarizes the productivity improvement due to heterogeneous ability.

More importantly, equation (14) implies that the magnitude of the distributional effect is captured by the amount of workers in the economy. As the large productivity gain from entrepreneurship brings about a large revenue gain, the weighted sum of productivity differences across firms are converted into the total sum of revenue differences across firms. On the other hand, large revenue differences mean that relatively talented entrepreneurs are stealing large amounts of demand from less able entrepreneurs. Hence, marginal entrepreneurs are forced to be workers. That 
is, the distributional effect influences aggregate productivity through the selection mechanism.

Substituting equation (14) into the distributional effect, real GDP can be rewritten as follows.

$$
Y=\left[\frac{1-\alpha}{\alpha}\right]^{\frac{1-\alpha}{\alpha}}\left[\exp x^{*}\left(\alpha, \sigma_{z}^{2}, \Gamma\left(N^{*}\right)\right)\right]\left[N^{*}\right]^{\frac{1}{\alpha}} .
$$

This equation suggests that real GDP is larger when the productivity of a marginal firm and the number of workers are large. The number of workers captures all distributional effects. It means that the impacts of heterogeneity on real GDP disappear once we control the number of workers.

\section{The Distribution of Entrepreneurial Ability}

In this section, we analyze how the distribution of entrepreneurial ability influences the firm size distribution and aggregate productivity. Without loss of generality, we assume that the population is equal to 1 and that ability is uniformly distributed. Suppose that $G(A)=\int_{A_{l}}^{A} \frac{1}{\gamma A_{l}} d a$. Then, $\Gamma(N)=(\gamma N+1) A_{l}$. The parameter $\gamma$ represents ability differences, while the parameter $A_{l}$ captures the overall level of ability. Real GDP and the equilibrium condition are rewritten as follows.

$$
\begin{aligned}
Y & =\left[\frac{1-\alpha}{\alpha}\right]^{\frac{1-\alpha}{\alpha}}\left[\exp x\left(\alpha, \sigma_{z}^{2},\left(\gamma N^{*}+1\right) A_{l}\right)\right]\left[N^{*}\right]^{\frac{1}{\alpha}}, \\
\frac{(1-\alpha) N^{*}}{\alpha} & =\int_{N^{*}}^{1} \exp \frac{\alpha\left[x\left(\alpha, \sigma_{z}^{2},(\gamma N+1) A_{l}\right)-x\left(\alpha, \sigma_{z}^{2},\left(\gamma N^{*}+1\right) A_{l}\right)\right]}{1-\alpha} d N .
\end{aligned}
$$

We assume that $\gamma>\left[\frac{2 \beta(1-\alpha)}{\sigma_{z}^{2} A_{l}}-1\right]$. This condition guarantees that the most able entrepreneurs have an incentive to processes information.

The following proposition can be proved. The formal proof is established in the Appendix. 
Proposition 2 Increases in both $\gamma$ and $A_{l}$ increase $Y$. On the other hand, while $N^{*}$ is clearly increasing in $\gamma$, the effect of $A_{l}$ on $N^{*}$ is ambiguous.

$$
N_{\gamma}\left(\alpha, \sigma_{z}^{2} ; \gamma, A_{l}\right)>0, \frac{d Y}{d \gamma}>0, \frac{d Y}{d A_{l}}>0
$$

The result shows that although increases in both $\gamma$ and $A_{l}$ improve the productivity of economy, only a change in $\gamma$ brings about a clear selection effect. When all agents improve their abilities, marginal entrepreneurs may be able to survive competition and remain as entrepreneurs due to their improved ability. Hence, $N_{A_{l}}\left(\alpha, \sigma_{z}^{2} ; \gamma, A_{l}\right)$ is ambiguous. However, an increase in $\gamma$ means that able entrepreneurs improve their ability more than less able entrepreneurs, and the marginal entrepreneurs are selected to be workers. Because of this selection effect, a change in $\gamma$ increases the average firm size and the average size differences.

\section{Conclusions and Extensions}

This paper models entrepreneurship as entrepreneur's information processing activity in order to predict changes in demand, and analyzes how entrepreneurship influences aggregate productivity and the distribution of firm size. We emphasize the productive impacts of competition and selection as a result of entrepreneurship.

Several extensions are considered. First, this paper emphasizes the importance of processing information for the adaptation to idiosyncratic shocks as a neglected role of entrepreneurship. Modeling firm entrepreneurship as information processing activity can address broader issues relating to entrepreneurship if the adjustment in input costs and persistent shocks are incorporated into the model. The broader issues that can be addressed include adopting a new technology, starting a new business, and developing a new product. These extensions are important so as to appreciate all aspects of entrepreneurship. 
Second, in this paper we assume that the distribution of ability is given. This is useful for simple analysis. However, as the literature on entrepreneurship suggests, ability can be acquired through previous experience and/or education. These investments change the future distribution of ability, while the expected future distribution of ability influences the incentive of entrepreneurs to invest. It would be interesting to examine what equilibrium is then obtained. This extension is also left for future research.

\section{Appendix}

The Proof of Theorem 1, and Proposition 1 and 2: Without loss of generality, we prove the theorem and propositions under the assumption of $N^{s}=1$ and $\Gamma(N)=$ $(\gamma N+1) A_{l}$. Define a function $B$ so that

$B\left(N^{*}, \alpha, \sigma_{z}^{2}, \gamma, A_{l}\right) \equiv \frac{1-\alpha}{\alpha} N^{*}-\int_{N^{*}}^{1} \exp \frac{\alpha\left[x\left(\alpha, \sigma_{z}^{2},(\gamma N+1) A_{l}\right)-x\left(\alpha, \sigma_{z}^{2},\left(\gamma N^{*}+1\right) A_{l}\right)\right]}{1-\alpha} d N$.

As $B\left(0, \alpha, \sigma_{z}^{2}, \gamma, A_{l}\right)<0, B\left(1, \alpha, \sigma_{z}^{2}, \gamma, A_{l}\right)>0$ and $\left.B_{N}\left(N^{*}, \alpha, \sigma_{z}^{2}, \gamma, A_{l}\right)\right|_{B\left(N^{*}, \alpha, \sigma_{z}^{2}, \gamma, A_{l}\right)=0}=$ $\frac{1}{\alpha}+x_{3}^{*}\left(\alpha, \sigma_{z}^{2},\left(\gamma N^{*}+1\right) A_{l}\right) \gamma A_{l} N^{*}>0$, the existence and uniqueness are obvious.

Note that

$$
N_{i}\left(\alpha, \sigma_{z}^{2}, \gamma, A_{l}\right)=-\frac{\left.B_{i}\left(N^{*}, \alpha, \sigma_{z}^{2}, \gamma, A_{l}\right)\right|_{B\left(N^{*}, \alpha, \sigma_{z}^{2}, \gamma, A_{l}\right)=0}}{\left.B_{N}\left(N^{*}, \alpha, \sigma_{z}^{2}, \gamma, A_{l}\right)\right|_{B\left(N^{*}, \alpha, \sigma_{z}^{2}, \gamma, A_{l}\right)=0}}, i=\alpha, \sigma_{z}^{2}, \gamma, A_{l} .
$$

Now, it is easy to derive that

$$
\begin{aligned}
\left.B_{\alpha}\left(N^{*}, \alpha, \sigma_{z}^{2}, \gamma, A_{l}\right)\right|_{B\left(N^{*}, \alpha, \sigma_{z}^{2}, \gamma, A_{l}\right)=0} & =-\frac{L^{*}}{\alpha}-F_{\alpha}, \\
\left.B_{i}\left(N^{*}, \alpha, \sigma_{z}^{2}, \gamma, A_{l}\right)\right|_{B\left(N^{*}, \alpha, \sigma_{z}^{2}, \gamma, A_{l}\right)=0} & =-F_{i}, i=\sigma_{z}^{2}, \gamma, A_{l},
\end{aligned}
$$


where

$$
\begin{gathered}
\alpha\left[\begin{array}{c}
x^{*}\left(\alpha, \sigma_{z}^{2},(\gamma N+1) A_{l}\right) \\
-x^{*}\left(\alpha, \sigma_{z}^{2},\left(\gamma N^{*}+1\right) A_{l}\right)
\end{array}\right] \\
F_{i}=\frac{\alpha}{1-\alpha} \int_{N^{*}}^{1} D_{i} \exp \frac{[-\alpha}{\partial i}, i=\alpha, \sigma_{z}^{2}, \gamma, A_{l} .
\end{gathered}
$$

Suppose that $N^{*} \leq \frac{1}{\gamma}\left[\frac{2 \beta(1-\alpha)}{\sigma_{z}^{2} A_{l}}-1\right]$. Then $x^{*}\left(\alpha, \sigma_{z}^{2},\left(\gamma N^{*}+1\right) A_{l}\right)=0$. Because we assume that $\gamma>\left[\frac{2 \beta(1-\alpha)}{\sigma_{z}^{2} A_{l}}-1\right]$, equation (8) implies that $D_{i}$ is positive for some $N$ where $i=\alpha, \sigma_{z}^{2}, \gamma, A_{l}$. Hence $N_{i}\left(\alpha, \sigma_{z}^{2}, \gamma, A_{l}\right)$ is positive for $i=\alpha, \sigma_{z}^{2}, \gamma, A_{l}$. Suppose that $N^{*}>\frac{1}{\gamma}\left[\frac{2 \beta(1-\alpha)}{\sigma_{z}^{2} A_{l}}-1\right]$. Equation (9) implies that $D_{\alpha}$ and $D_{\sigma_{z}^{2}}$ are still positive. Hence, $N_{\alpha}\left(\alpha, \sigma_{z}^{2}, \gamma, A_{l}\right)$ and $N_{\sigma_{z}^{2}}\left(\alpha, \sigma_{z}^{2}, \gamma, A_{l}\right)$ are positive. We need to check $D_{\gamma}$ and $D_{A_{l}}$.

$$
\begin{aligned}
\frac{\partial x^{*}\left(\alpha, \sigma_{z}^{2},(\gamma N+1) A_{l}\right)}{\partial \gamma \partial N} & =\frac{\beta\left\{\left(1-\frac{\gamma N}{2}\right) \sqrt{\frac{\sigma_{z}^{2}(\gamma N+1) A_{l}}{2 \beta(1-\alpha)}}-(1-\gamma N)\right\}}{(\gamma N+1)^{3} A_{l}^{2}} \\
& \geq \frac{\beta\left[\left(1-\frac{\gamma N}{2}\right)-(1-\gamma N)\right]}{(\gamma N+1)^{3} A_{l}^{2}}=\frac{\beta \gamma N}{2(\gamma N+1)^{3} A_{l}^{2}}>0 .
\end{aligned}
$$

Hence, $D_{\gamma}$ and $N_{\gamma}\left(\alpha, \sigma_{z}^{2}, \gamma, A_{l}\right)$ are also positive. On the other hand, as $\frac{\partial x^{*}\left(\alpha, \sigma_{z}^{2},(\gamma N+1) A_{l}\right)}{\partial A_{l} \partial N}$ can be positive or negative, $N_{A_{l}}\left(\alpha, \sigma_{z}^{2}, \gamma, A_{l}\right)$ is ambiguous.

Because $N_{\gamma}\left(\alpha, \sigma_{z}^{2}, \gamma, A_{l}\right)>0, \frac{d Y}{d \gamma}>0$. We need to check the sign of $\frac{d Y}{d A_{l}}$.

$$
\begin{aligned}
\frac{d Y}{d A_{l}} & =\frac{1}{\alpha}\left[\frac{1-\alpha}{\alpha}\right]^{\frac{1-\alpha}{\alpha}}\left[\exp x^{*}\left(\alpha, \sigma_{z}^{2},\left(\gamma N^{*}+1\right) A_{l}\right)\right]\left(N^{*}\right)^{\frac{1}{\alpha}-1} X_{A_{l}}, \\
X_{A_{l}} & =\alpha \frac{\partial x^{*}\left(\alpha, \sigma_{z}^{2},\left(\gamma N^{*}+1\right) A_{l}\right)}{\partial A_{l}} N^{*}+\left[1+\alpha \frac{\partial x^{*}\left(\alpha, \sigma_{z}^{2},\left(\gamma N^{*}+1\right) A_{l}\right)}{\partial N^{*}} N^{*}\right] N_{A_{l}}\left(\gamma, A_{l}, \sigma_{z}^{2}, \alpha\right) .
\end{aligned}
$$

As it is shown that $X_{A_{l}}>0, \frac{d Y}{d A_{l}}>0$. Q.E.D.

\section{References}

[1] Blackwell, D. (1953), "Equivalent Comparisons of Experiments," Annals of Mathematical Statistics, Vol.24, 265-272. 
[2] Casson, Mark (2005), "Entrepreneurship and the Theory of the Firm," Journal of Economic Behavior and Organization, Vol.58, 327-348.

[3] Fiet, James O. (1996), "The Informational Basis of Entrepreneurial Discovery," Small Business Economics Vol.8, 419-430.

[4] Hassler, John and Mora, Jose V., Rodriguez (2000), "Intelligence, Social Mobility, and Growth" American Economic Review, Vol.90, 888-908.

[5] Hayek, Friedrich (1945), "The Use of Knowledge in Society," American Economic Review, Vol.35, 519-530.

[6] Hey, John D. (1979), Uncertainty in Microeconomics, Martin Robertson \& Company Ltd., Oxford.

[7] Kirzner (1973), Competition and Entrepreneurship, University of Chicago Press, Chicago.

[8] Kirzner, Israel M. (1997), " Entrepreneurial Discovery and the Competitive Market Process: An Austrian Approach," Journal of Economic Literature, Vol.35, $60-83$.

[9] Knight, Frank (1921), Risk, Uncertainty and Profit, Houghton Mifflin Co., New York.

[10] Mills, David E. and Schumann, Laurence (1985), "Industry Structure with Fluctuating Demand," American Economic Review, Vol.75, 758-767.

[11] Rothschild, Michael and Stiglitz, Joseph E. (1970), "Increasing Risk: 1. A Definition," Journal of Economic Theory, Vol.2, 225-243.

[12] Schultz, Theodore W. (1975), "The Value of the Ability to Deal with Disequilibria," Journal of Economic Literature, Vol.13, 827-846. 
[13] Schumpeter, Joseph (1947), "The Creative Response in Economic History," Journal of Economic History, Vol.7, 149-159.

[14] Shane, Scott (2000), "Prior Knowledge and the Discovery of Entrepreneurial Opportunities," Organization Science, Vol.11, 448-469.

[15] Takii, Katsuya (2003), "Prediction Ability," Review of Economic Dynamics, Vol.6, 80-98.

[16] Takii, Katsuya (2004), "Entrepreneurial Efficiency: Theory," OSIPP Discussion Paper: DP-2004-E-005, November.

[17] Takii, Katsuya (2007), "The Value of Adaptability - Through the Analysis of a Firm's Prediction Ability," Journal of Economics and Business, Vol.59, 144-162.

[18] Takii, Katsuya (2008), "Fiscal Policy and Entrepreneurship," Journal of Economic Behavior and Organization, Vol.65, 592-608.

[19] Thesmar, David and Thoenig, Mathias (2000), Creative Destruction and Firm Organization Choice, Quarterly Journal of Economics, Vol.115, 1201-1237.

[20] Welch, F. (1970), Education in Production, Journal of Political Economics, Vol.78, 35-59. 